\title{
RELATIONSHIP BETWEEN PROP (6-n-PROPYLTHIOURACIL) TASTER STATUS AND PREFERENCE FOR DIFFERENT TASTE FOOD GROUPS AMONG UNIVERSITY STUDENTS
}

\author{
SYATHIRAH HANIM, A.H. ${ }^{1}$, RUHAYA, H. ${ }^{1}$, NORKHAFIZAH, S. ${ }^{1}$ and MARINA, A.M..$^{2 *}$ \\ ${ }^{1}$ School of Dental Sciences, Health Campus, Universiti Sains Malaysia, \\ 16150 Kubang Kerian, Kelantan, Malaysia \\ ${ }^{2}$ Nutrition and Dietetic Programme, School of Health Sciences, Health Campus, \\ Universiti Sains Malaysia, 16150 Kubang Kerian, Kelantan, Malaysia \\ "E-mail:mareena@usm.my
}

Accepted 10 October 2020, Published online 31 December 2020

\begin{abstract}
It is reported by many studies that supertasters are highly sensitive towards strong taste such as bitter, and therefore had a lower preference for those foods. However, whether the findings apply to all cultures is still debated. The study on the Malaysian population regarding their PROP status is scarce. Thus, we carried out a study to determine whether 6-npropylthiouracil (PROP) sensitivity status affects the food preference among university students in Kelantan, Malaysia. PROP taster status was determined using PROP test paper and food preference was determined using a questionnaire. The results indicated that there was no significant difference $(p>0.05)$ in the food preference between supertasters, medium tasters, and non-tasters except for jelly, dark chocolate, and plain tea. Contrary to popular belief that PROP taster status was negatively associated with a bitter taste, the present study found a positive correlation between PROP scores and few foods from the bitter food group (bitter gourd, dark chocolate, and plain tea) and also sweet food group (chocolate cake and chocolate spread). This indicates that the genetic factor that is associated with PROP sensitivity does not play important role in determining the food preference among Malaysians. Instead, other factors such as culture and environment could be more influential in defining the way Malaysians select their foods.
\end{abstract}

Key words: Food preference; PROP; taster; taste sensitivity

\section{INTRODUCTION}

The taste of food plays a major role in determining the food preference of an individual. The different preference over food varies across individuals and ethnics and define us of who are. As health is very much influence by food intake and affects the choice of food taken, thus food does have a great impact on the health outcome. Question on what makes us prefer and enjoy certain meals and reject others is always of interest. Taste perception has great importance in governing the choice of foods we take. Each taste gives a meaningful message to the brain to ascertain we obtain the right type of food and avoid the harmful substance that can pose a hazard. For example, the sweet flavor is important to make sure that we are getting enough caloric

\footnotetext{
* To whom correspondence should be addressed.
}

energy contributed by sugars and carbohydrates, while the bitter taste warns us of a possible poisoning food (Bradbury, 2014).

Phenylthiocarbamide (PTC) and its chemically related compound, 6-n-propylthiouracil (PROP), provide extremely bitter taste to some subjects (tasters) but are tasteless or only slightly bitter to others (non-tasters) (Catanzaro et al., 2013). Due to the slightly toxic effect of PTC, PROP is generally used to determine the status of taste sensitivity of individuals. The term non-taster, medium taster, and supertaster were given to individuals based on their ability to taste PROP. Non tasters have two recessive alleles ( $t t)$, and they perceive PROP the least. Supertasters have two dominant alleles (TT), and these individuals perceive PROP the most. Medium tasters are thought to have one dominant and one recessive allele $(\mathrm{Tt})$, and they have an intermediate degree between non-tasters and supertasters 
(Barthoshuk, 1994). The bitter receptor gene has been identified as TAR2R38, which is located on chromosome 7p35 in the human genome (Kim et al., 2003).

Since PROP sensitivity has a direct influence on the dietary pattern, thus, it is thought to influence the non-communicable disease such as obesity and diabetes in the general population. Previous studies have established an association between PROP sensitivity and various food intake, food preference, and body weight (Pasquet et al., 2002; Tepper, 2008; Catanzaro et al., 2013). Some reported PROP tasters show lower acceptance of cruciferous and other bitter vegetables and fruits (Drewnowski et al., 2000). Thus, these individuals could have deprived intake of important antioxidant phytochemicals that contained cancer-preventing effects.

It is established by previous research that the PROP taster status of individuals (whether nontaster, medium taster, or supertaster) influence their selection of food. Medium and supertasters are said to perceive more intense bitterness and other taste sensations than do non-tasters, and therefore dislike foods that are bitter, spicy, or have strong tasting (a distinctive flavor that tends to linger on the tongue). On the contrary, non-taster was reported to prefer bitter and strong-tasting food. However, the results were mixed and some studies did not establish any relationship between the PROP taster status and food preference (Pasquest et al., 2002; Keller \& Tepper, 2004; Tepper et al., 2009). The report regarding food preference and its relationship with PROP sensitivity is scarce among Malaysians. A study by Ooi et al. (2010), reported that the bitter receptor gene could not predict the aversion of sweet and bitter vegetables among Malaysian subjects. However, only vegetables with sweet and bitter taste were included in the study. Thus, the present was conducted to determine the relationship between PROP sensitivity status and preference for various food groups such as sweet, sour, salty, and bitter taste.

\section{MATERIALS \& METHODS}

\section{Subjects}

This is a cross-sectional study whereby participants were selected by convenience sampling. Power and Sample Size Calculation (PS) software were used to calculate the number of sample size in this study. A total of 158 undergraduate students (age between 19 to 25) in Kelantan state, participated in this study. University students were chosen as subjects in the present study as they represent the youth generation in Malaysia. The inclusion criteria were undergraduate students and free from any disease or condition that can alter taste sensation. Subjects were excluded if they were a smoker or taking long term medication for chronic disease. The research procedure was approved by the Ethical Research Committee, Universiti Sains Malaysia (USM/ JEPeM/18070313).

\section{PROP taster classification method}

Taste sensitivity was obtained by using the PROP test paper ( $3 \mathrm{~cm}$ length) to detect the perception of the bitter substrate. A PROP taste paper (PL Precision, Northampton), with 20 micrograms of PROP concentration was placed on the tongue of the subjects for $30 \mathrm{sec}$. First, subjects were given instruction on how to use Green's Labeled (LMS) according to the method by Green et al. (1993). The scale of the Green LMS was ranged from 0 to 100 with 0 represent barely detectable sensation and 100 represent the strongest imaginable sensation of any kind. Then, subjects were asked to rate the degree of sensation perceived (intensity of the bitterness) accordingly. The subjects were identified as non-taster, medium taster, and supertaster if they scored $<12 \mathrm{mM}, 12-60 \mathrm{mM}$, and $>60 \mathrm{mM}$ respectively.

\section{Food preference}

Food preference was determined by using a food preference questionnaire. The list of food items that represent the taste of sweet, sour, salty, and bitter was developed based on previous questionnaires (Drewnoski \& Gomez-Carneros, 2000; Ooi et al., 2000; Choong et al., 2012; Tornwall et al., 2012; Asano et al., 2016; Amarra et al., 2016; Jiet \& Soma 2017; Carbonneau, 2017). The food item lists were chosen to suit the Malaysian population. The questionnaire consisted of a total of 53 food items. Subjects were required to indicate their preference towards the foods on a 7-point hedonic scale, from 'dislike extremely' to 'like extremely'. Content validity was assessed by expert panels by reviewing and commenting on the questionnaire and the food list items.

\section{Statistical analysis}

All data collected were entered and analyzed using IBM Statistical Package of Social Science (SPSS) version 24.0. A one-way ANOVA test was used to determine the significant difference for food preference among non-taster, medium taster, and supertaster group. PROP bitterness rating was correlated with food preference score by using Spearman's rank correlation. $p<0.05$ was considered as statistically significant. 


\section{RESULTS}

Demographic characteristics and taster status

A total number of 158 participants of undergraduate students were recruited in this study. Table 1 shows an overview of the demographic characteristics of the participants. Most participants were female with a total of 126 students $(79.7 \%)$ and 32 male students $(20.3 \%)$. Most of the participants were Malay (79.1\%), followed by Chinese $(10.8 \%)$, other ethnics minorities $(5.7 \%)$, and Indians (4.4\%).

\section{Food preference}

Table 2 shows the mean food preference scores among non-tasters, medium tasters, and supertasters. There were 17 types of food categorized under the sweet food group. The mean food preference scores among non-tasters, medium tasters, and supertasters were not significantly different $(p>0.05)$ for all items in the sweet food group except for jelly $(p=0.024)$. The mean food preference score for all 15 items in the salty food group among non-tasters, medium tasters, and supertasters was not significantly different $(p>0.05)$. A similar finding was observed for all 9 food items in the sour food group.

There were 12 foods listed under the bitter group and most were not significantly different $(p>0.05)$ among non-tasters, medium tasters, and supertasters except for dark chocolate and plain tea. There was a significant difference $(p<0.05)$ between non-tasters and supertasters in the food preference score for dark chocolate, in which supertasters had a significantly higher preference score for dark chocolate than did non-tasters. The result for plain tea showed that there was a significant difference $(p<0.05)$ in the mean food preference score between medium tasters and supertasters, which also indicated a higher preference of supertasters towards plain tea than did medium tasters.

Under the sweet food group category, there was a significant correlation between PROP bitterness rating with chocolate cake $(r=0.185)$ and chocolate spread $(r=0.194)$. There was no correlation between taste PROP bitterness rating and preference for all food items in the sour group. The soup was the only food under the salty food group which had a negative significant correlation with PROP scores $(r=-0.1 .33)$. The foods under the bitter food group that had significant positive correlations with PROP scores were bitter gourd $(r=0.165)$, dark chocolate $(r=0.194)$, and plain tea $(r=0.155)$.

\section{DISCUSSION}

The current study indicated that supertasters constituted the highest group among the subjects. This indicated that there were more tasters than nontasters, which supported a study by Ooi et al. (2010), which found that the non-tasters group consisted of the lowest number when tested by determining the bitter receptor gene TAS2R38, among Malaysian subjects. However, the result of the present study contradicts the study by Tepper (2009), which indicated that the percentage of only $20 \%$ of supertasters, $50 \%$ of medium tasters, and $30 \%$ of non-tasters. Nevertheless, the present study was also supported by the study of Karmatakar (2016) among 6 to 12-year-old schoolchildren. The result showed that the supertasters group (34.8\%) was the highest, followed by non-tasters (34\%), and medium tasters (31.2\%) group.

Many studies recorded medium taster as being the highest number in their samples. For example, a study by Gunawardane et al. (2016) among 78 healthy university students in Sri Lanka found that medium taster (37.18\%) was the largest group, followed by supertaster $(34.62 \%)$ and non-taster (28.21\%). Similar to Gunawardane's findings, Alsafi and Diab (2017) also recorded that the majority of their samples were medium tasters (44.12\%), followed by supertasters $(34.71 \%)$ and non-tasters $(21.18 \%)$.

Table 1. Demographic characteristics of non-tasters, medium tasters, and supertasters

\begin{tabular}{lcccr}
\hline & $\begin{array}{c}\text { Non-tasters } \\
(n=37)\end{array}$ & $\begin{array}{c}\text { Medium tasters } \\
(n=34)\end{array}$ & $\begin{array}{c}\text { Supertasters } \\
(n=87)\end{array}$ & $\begin{array}{c}\text { Total } \\
(n=158)\end{array}$ \\
\hline Race & & & & \\
$\quad$ Malay & $28(22.4)$ & $27(21.6)$ & $70(56.0)$ & 125 \\
$\quad$ Chinese & $3(17.6)$ & $4(23.5)$ & $10(58.8)$ & 17 \\
$\quad$ Indians & $3(42.9)$ & $1(14.2)$ & $3(42.9)$ & 7 \\
Others & $3(33.3)$ & $2(22.2)$ & $2(22.2)$ & 9 \\
Gender & & & & \\
$\quad$ Male & $8(25.0)$ & $9(28.1)$ & $15(46.9)$ & 32 \\
Female & $29(23.0)$ & $25(19.8)$ & $72(57.1)$ & 126 \\
\hline
\end{tabular}

Numbers in parentheses indicate percentage. 
Table 2. Mean food preference score among non-taster, medium taster, and supertaster $(n=158)$ and correlations of PROP score and food preference

\begin{tabular}{|c|c|c|c|c|c|}
\hline \multirow{2}{*}{ Food Groups } & \multicolumn{4}{|c|}{ Mean (SD) } & \multirow{2}{*}{$\begin{array}{l}\text { Correlations o } \\
\text { PROP score } \\
\text { and food } \\
\text { preference }(r)\end{array}$} \\
\hline & $\begin{array}{c}\text { Non-tasters } \\
(n=37)\end{array}$ & $\begin{array}{l}\text { Medium tasters } \\
\quad(n=34)\end{array}$ & $\begin{array}{l}\text { Supertasters } \\
\qquad(n=87)\end{array}$ & $p$ value & \\
\hline \multicolumn{6}{|l|}{ Sweet Food } \\
\hline Chocolate Bar & $5.35 \pm 1.50$ & $5.50 \pm 1.31$ & $5.63 \pm 1.44$ & 0.596 & 0.082 \\
\hline Baby Corn & $4.70 \pm 1.58$ & $4.71 \pm 1.45$ & $4.83 \pm 1.63$ & 0.888 & 0.036 \\
\hline Candy & $4.92 \pm 1.44$ & $4.71 \pm 1.59$ & $4.92 \pm 1.41$ & 0.751 & 0.012 \\
\hline Chocolate cake & $5.30 \pm 1.73$ & $5.68 \pm 1.34$ & $5.95 \pm 1.33$ & 0.066 & $0.185^{\star}$ \\
\hline Chocolate spread & $4.97 \pm 1.85$ & $5.06 \pm 1.59$ & $5.66 \pm 1.38$ & 0.103 & $0.194^{*}$ \\
\hline Chocolate syrup & $4.68 \pm 1.68$ & $4.97 \pm 1.72$ & $4.92 \pm 1.65$ & 0.575 & 0.061 \\
\hline Cookies & $5.59 \pm 1.30$ & $5.38 \pm 1.35$ & $5.87 \pm 1.22$ & 0.137 & 0.114 \\
\hline Doughnut & $5.41 \pm 1.30$ & $5.18 \pm 1.40$ & $5.39 \pm 1.37$ & 0.708 & 0.009 \\
\hline Honey & $4.97 \pm 1.54$ & $4.76 \pm 1.52$ & $5.09 \pm 1.50$ & 0.563 & 0.047 \\
\hline Ice-cream & $6.08 \pm 1.21$ & $5.47 \pm 1.24$ & $6.02 \pm 1.21$ & 0.055 & 0.019 \\
\hline Jam & $4.81 \pm 1.31$ & $4.29 \pm 1.32$ & $4.71 \pm 1.54$ & 0.263 & -0.001 \\
\hline Jelly & $5.08 \pm 1.45^{a}$ & $4.35 \pm 1.07^{b}$ & $5.06 \pm 1.36^{a}$ & 0.024 & 0.036 \\
\hline Marshmallow & $4.41 \pm 1.64$ & $4.24 \pm 1.58$ & $4.51 \pm 1.75$ & 0.729 & 0.036 \\
\hline Milk Chocolate & $5.27 \pm 1.69$ & $5.68 \pm 1.53$ & $5.46 \pm 1.71$ & 0.593 & 0.032 \\
\hline Muffin/Cupcake & $5.41 \pm 1.55$ & $5.12 \pm 1.45$ & $5.54 \pm 1.39$ & 0.352 & 0.049 \\
\hline Popcorn Caramel & $5.30 \pm 1.43$ & $5.21 \pm 1.04$ & $5.25 \pm 1.62$ & 0.966 & -0.009 \\
\hline Popsicles & $5.05 \pm 1.60$ & $4.41 \pm 1.67$ & $4.84 \pm 1.53$ & 0.219 & -0.028 \\
\hline \multicolumn{6}{|l|}{ Salty Food } \\
\hline Cheddar cheese & $5.49 \pm 1.73$ & $5.47 \pm 1.44$ & $5.49 \pm 1.41$ & 0.997 & 0.003 \\
\hline Chicken nugget & $6.03 \pm 1.50$ & $5.62 \pm 1.33$ & $5.87 \pm 1.17$ & 0.401 & -0.028 \\
\hline Corn snacks & $4.84 \pm 1.50$ & $4.50 \pm 1.29$ & $4.72 \pm 1.34$ & 0.569 & -0.018 \\
\hline Dried anchovies & $4.54 \pm 1.63$ & $4.32 \pm 1.41$ & $4.39 \pm 1.56$ & 0.825 & -0.033 \\
\hline Dried cuttlefish & $3.95 \pm 1.86$ & $3.91 \pm 1.64$ & $3.63 \pm 1.74$ & 0.567 & -0.081 \\
\hline Dried prawns & $3.86 \pm 1.55$ & $3.65 \pm 1.79$ & $3.51 \pm 1.63$ & 0.539 & -0.089 \\
\hline Dried salted fish & $3.92 \pm 1.92$ & $3.65 \pm 1.70$ & $3.89 \pm 1.80$ & 0.775 & 0.004 \\
\hline Fried noodles & $5.43 \pm 1.43$ & $5.00 \pm 1.61$ & $5.02 \pm 1.60$ & 0.368 & -0.097 \\
\hline Fried rice & $5.65 \pm 1.14$ & $5.41 \pm 1.08$ & $5.61 \pm 1.40$ & 0.692 & 0.001 \\
\hline Noodles with soup & $6.14 \pm 1.08$ & $5.53 \pm 1.31$ & $5.69 \pm 1.38$ & 0.115 & -0.117 \\
\hline Noodles with soy sauce & $4.97 \pm 1.40$ & $4.85 \pm 1.33$ & $5.13 \pm 1.49$ & 0.619 & 0.056 \\
\hline Potato chips & $5.32 \pm 1.65$ & $5.35 \pm 1.23$ & $5.54 \pm 1.24$ & 0.640 & 0.072 \\
\hline Prawn crackers & $4.78 \pm 1.67$ & $4.62 \pm 1.58$ & $4.68 \pm 1.50$ & 0.899 & -0.022 \\
\hline Salted squids & $3.70 \pm 2.05$ & $3.32 \pm 1.53$ & $3.60 \pm 1.91$ & 0.674 & -0.009 \\
\hline Soup & $6.27 \pm 0.93$ & $5.62 \pm 1.18$ & $5.79 \pm 1.33$ & 0.058 & $-0.133^{*}$ \\
\hline \multicolumn{6}{|l|}{ Sour Food } \\
\hline Blueberry flavour & $5.19 \pm 1.22$ & $4.71 \pm 1.40$ & $4.86 \pm 1.38$ & 0.295 & -0.081 \\
\hline Cranberry flavour & $4.65 \pm 1.60$ & $4.44 \pm 1.16$ & $4.48 \pm 1.40$ & 0.788 & -0.042 \\
\hline Grapefruit & $5.70 \pm 1.49$ & $5.59 \pm 1.54$ & $5.80 \pm 1.34$ & 0.744 & 0.040 \\
\hline Lemon & $4.54 \pm 1.79$ & $4.71 \pm 1.14$ & $4.80 \pm 1.49$ & 0.669 & 0.071 \\
\hline Orange & $5.68 \pm 1.44$ & $5.62 \pm 1.30$ & $5.84 \pm 1.22$ & 0.640 & 0.062 \\
\hline Red currant juice & $4.95 \pm 1.45$ & $4.41 \pm 1.62$ & $4.48 \pm 1.33$ & 0.193 & -0.117 \\
\hline Sour milk & $3.62 \pm 2.18$ & $3.71 \pm 1.68$ & $3.21 \pm 1.86$ & 0.323 & -0.103 \\
\hline Strawberry & $5.65 \pm 1.42$ & $5.12 \pm 1.41$ & $5.32 \pm 1.68$ & 0.350 & -0.076 \\
\hline Yogurt & $6.03 \pm 1.26$ & $5.65 \pm 1.41$ & $5.87 \pm 1.35$ & 0.490 & -0.029 \\
\hline \multicolumn{6}{|l|}{ Bitter Food } \\
\hline Bitter gourd & $2.43 \pm 1.46$ & $2.97 \pm 1.77$ & $3.21 \pm 2.04$ & 0.109 & $0.165^{*}$ \\
\hline Broccoli & $5.30 \pm 1.71$ & $5.47 \pm 1.56$ & $5.21 \pm 1.67$ & 0.734 & -0.034 \\
\hline Cabbage & $5.57 \pm 1.17$ & $5.50 \pm 1.56$ & $5.46 \pm 1.45$ & 0.927 & -0.031 \\
\hline Cauliflower & $5.62 \pm 1.26$ & $5.41 \pm 1.69$ & $5.63 \pm 1.40$ & 0.736 & 0.016 \\
\hline Chinese kale & $5.38 \pm 1.96$ & $4.91 \pm 1.98$ & $4.98 \pm 1.60$ & 0.447 & -0.082 \\
\hline Coffee & $4.89 \pm 1.79$ & $4.62 \pm 1.67$ & $4.82 \pm 1.91$ & 0.806 & -0.007 \\
\hline Cucumber & $4.68 \pm 1.87$ & $5.03 \pm 1.55$ & $5.24 \pm 1.36$ & 0.173 & -0.148 \\
\hline Dark chocolate & $4.43 \pm 1.91^{b}$ & $4.94 \pm 1.54^{b}$ & $5.25 \pm 1.65^{a}$ & 0.049 & $0.194^{*}$ \\
\hline Eggplant & $4.11 \pm 1.94$ & $3.71 \pm 1.88$ & $4.20 \pm 1.81$ & 0.425 & 0.039 \\
\hline French bean & $4.62 \pm 1.69$ & $4.85 \pm 1.64$ & $4.63 \pm 1.54$ & 0.769 & -0.009 \\
\hline Lady's fingers & $4.92 \pm 2.14$ & $4.82 \pm 1.95$ & $4.92 \pm 1.88$ & 0.969 & -0.004 \\
\hline Plain tea & $4.08 \pm 1.71^{\mathrm{a}}$ & $3.68 \pm 1.30^{\mathrm{b}}$ & $4.45 \pm 1.85^{\mathrm{a}}$ & 0.021 & $0.155^{*}$ \\
\hline
\end{tabular}

Values presented are means \pm SD.

$p$-value in bold indicates a significant difference $(p<0.05)$ with different letters in a row shows significant differences.

$r$ value with $\left(^{*}\right)$ indicates a significant difference at $p<0.05$. 
The variation in the prevalence of PROP taster status among the population occurred due to different age span discrepancies and geographic distributions. It may also be attributed to the different cut-off scores for grouping taste status. Hence, exact comparisons with previous studies are not feasible. Green (1993) suggested the usage of bitterness intensity on the general Labelled Magnitude scale (LMS) which later on can be categorized into several groups; (1) supertasters $(>60)$. (2) medium tasters (12-60), and (3) non-tasters $(<12)$. However, there was also a modified cut-off scale that was used by Goldstein et al. (2007) and Jyothirmai et al. (2011) in which the cut-off score was as follows: (1) supertasters $(>67),(2)$ medium tasters (15 to 67), and (3) non-tasters $(<15)$. Therefore, the different varieties of cut off points resulted in different grouping in taster status.

In general, the present study recorded no significant difference $(p>0.05)$ in the preference for all food groups except for jelly (sweet), dark chocolate (bitter group), and plain tea (bitter group) among non-tasters, medium tasters, and supertasters. The present study found that the sour and salty food group did not have much impact on the taste sensitivity status among subjects. None of the groups had any significant difference among nontasters, medium tasters, and supertasters. Only one food (soup) shows the correlation for salty taste while none for the sour group. The supertasters did not prefer much soup, which is shown by the negative correlation. Although not significant, the sour and salty groups did have quite a several negative correlations with the PROP bitterness rating. However, a study by Borazon et al. (2012) found that supertasters had the greatest preference for condiments, which were mostly salty and sour. This indicates that food preference of supertasters varies across cultural and genetic factor does not play a strong role in determining their preference for a certain taste.

In terms of the sweet food category, the present study found that chocolate cake and chocolate spread were positively correlated with PROP taste sensitivity. However, chocolate can be both sweet and bitter, depending on the individual's perception. We put these foods under the sweet category since the sweetness seems to mask the bitter taste of chocolate.

The findings from previous studies suggested that supertasters were more likely to reject bitter food more so than non-tasters (Kaminski et al., 2000). The present study however does not support the findings. Contrary to the expected negative direction, a positive correlation between the dark chocolate (bitter food group) with PROP scores was found. Surprisingly, the preference for dark chocolate was also significantly preferred $(p<0.05)$ by the supertasters as compared to the non-tasters.

The present study also found a positive significant relationship between PROP bitterness rating and plain tea, another bitter food, whereby a significant difference $(p<0.05)$ was found between the medium taster and supertaster group. Our result conflicts with those of Akella et al. (1997), which found lower acceptance of green tea linked to genetic PROP sensitivity. Our study supports a study by Ooi et al. (2010) which found that non-tasters dislike green tea more than tasters, which was conducted on Malaysian subjects. Other bitter foods found to be positively associated with PROP scores in the present study were bitter gourd, cucumber, and plain tea. The present study suggested that super taster can appreciate bitter food. According to Catanzaro et al. (2013), bitter and spicy food maybe be perceived as enjoyable even to medium taster or supertaster.

We hypothesized that the genetic factor for bitterness, which is indicated by PROP taster status does not influence much the food preference for subjects in this study. Most of the listed foods were generally accepted and familiar among the subjects. In Malaysia, most of the vegetables that represent the bitter food group are not eaten raw but mostly eaten in the cooked form to reduce bitterness. For example, bitter gourd, which is known as a bitter vegetable, is commonly cooked with egg and some seasoning added to balance the bitter taste. Therefore, another possible description for our finding is that the culinary aspect had overshadowed the bitter vegetable acceptance as suggested by Mattes (2004). Drinking tea and coffee is common in Malaysian culture among Malays, Chinese, Indians, and other ethnic groups. However, tea and coffee were commonly consumed by Malaysians with added sugar or sweetened condensed milk (Amarraet et al., 2016). It is no surprise if the sugar was added more than enough to mask the bitter taste. As indicated by Norliza-Ahmad et al. (2019), undergraduate students in Malaysia were found to have a high prevalence of sugar-sweetened beverage consumption. Hence, the items such as tea and coffee could not purely show the subjects' preference towards the bitter taste.

Also, the abundance of food products in the market and the advertisement of food in the media play an important role by leading the changes of taste acceptance and food preferences among the participants (Lanfer et al., 2013). The coffeedrinking culture among the young generation is common nowadays and coffee shops and cafes have become the favorite social meeting place for them. 
Thus, it is possible that supertasters that supposedly reject the bitter taste of coffee would soon learn to adapt to it due to influence from peers.

In general, our study supports the study by Catanzaro et al. (2013), which pointed out that the role of culture in shaping preference towards food is more influential than the genetic factors. Our study also supports the study by Ooi et al. (2010) which found that the bitter receptor gene TAS2R38 was not a suitable predictor of food selection among Malaysians.

\section{CONCLUSION}

Early researchers suggested that taste sensitivity influences the guiding of food preferences, such that tasters had more overall food dislikes than nontasters. However, the results of the present study do not support the idea. We found that the relationship exists in the opposite direction. Supertasters may be sensitive to sweet and bitter taste than do nontasters, but it does not necessarily mean that they detest the taste. Other factors other than genetic such as culture and environment could have more weight in determining the food preference of university students in the present study.

\section{ACKNOWLEDGEMENT}

This study was supported by Universiti Sains Malaysia, under the Research University (RU) grant (1001/PPSK/8012270).

\section{REFERENCES}

Akella, G.D., Henderson, S.A. \& Drewnowski, A. 1997. Sensory acceptance of Japanese green tea and soy products is linked to genetic sensitivity to 6-n-propylthiouracil. Nutrition and Cancer, 29(2): 146-151.

Alsafi, R.A. \& Diab, B.S. 2017. Impact of bitter taste threshold on caries experience in relation to ABO blood types among dental students at Al Kufa University. Journal of Baghdad College of Dentistry, 29(3): 121-127.

Amarra, M.S.V., Khor, G.L. \& Chan, P. 2016. Intake of added sugar in Malaysia: a review. Asia Pacific Journal of Clinical Nutrition, 25(2): 227-240.

Asano, M., Hong, G., Matsuyama, Y., Wang, W., Izumi, Y., Toda, T. \& Kudo, T.A. 2016. Association of oral fat sensitivity with body mass index, taste preference and eating habit in healthy Japanese young adults. Tohuku Journal Experimental Medicine, 238: 93-103.
Bartoshuk, L.M., Duffy, V.B. \& Miller, I.J. 1994. PTC/PROP tasting: Anatomy, psychophysics, and sex effects. Physiology \& Behavior, 56(6): 1165-1171.

Borazon, E.Q., Villarino, B.J., Magbuhat, R.M.T. \& Sabandal, M.L. 2012. Relationship of PROP (6-n-propylthiouracil) taster status with body mass index, food preferences, and consumption of Filipino adolescents. Food Research International, 47(2): 229-235.

Bradbury, J. 2004. Taste perception: cracking the code. PLOS Biology, 2(3): 0295-0297.

Carbonneau, E., Bradette-Laplante, M., Lamarche, B., Provencher, V., Bégin, C., Robitaille, J. \& Lemieux, S. 2017. Development and validation of the food liking questionnaire in a FrenchCanadian population. Nutrients, 9(12): 1337(110).

Catanzaro, D., Chesbro, E.C. \& Velkey, A.J. 2013. Relationship between food preference and PROP taster status of college students. Appetite, 68: 124-131.

Choong, S.SY., Balan, S.N., Chua, L.S. \& Say, Y.H. 2012. Preference and intake frequency of high sodium foods and dishes and their correlations with anthropometric measurements among Malaysian subjects. Nutrition Research and Practice, 6(3):238-245.

Drewnowski, A., Handerson, S.A., Han, S.C., Berg, W.A. \& Ruffin, M.T. 2000. Genetic taste markers and preferences for vegetables and fruit of female breast cancer patients. Journal of the American Dietetic Association, 100(2): 191-197.

Drewnowski, A. \& Gomez-Carneros, C. 2000. Bitter taste, phytonutrients, and the consumer: a review. American Journal of Clinical Nutrition, 72(6): 1424-1435.

Goldstein, G., Daun, H. \& Tepper, B. 2007. Influence of PROP taster status and maternal variables on energy intake and body weight of preadolescents. Physiology Behaviour, 90: 809-817.

Green, B.G., Shaffer, G.S. \& Gilmore, M.M.A. 1993. Semantically labelled magnitude scale of oral sensation with apparent ratio properties. Chemical Senses, 18: 683-702.

Gunawardane, S., Ariyasinghe, S. \& Rajapakse, S. 2016. Study of the relationship between taste sensation and dental caries experience among dental students. Indian Journal of Oral Sciences, 7(2): 99-102.

Jiet, L.J. \& Mitra Soma, R. 2017. High salt diets in young univesity adults and the correlation with blood pressure, protein intake and fat free mass. Bioscience Horizons, 10: 1-13.

Jyothirmai, J., Naganandini, S. \& Aradhya, S. 2011. Caries experience in 15-year-old school children in Bangalore with inherited taste sensitivity levels to 6-n-propylthiouracil: an observational 
study. Journal of Investigative and Clinical Dentistry, 2(1): 51-56.

Kaminski, L.C., Henderson, S.A. \& Drewnowski, A. 2000. Young women's food preferences and taste responsiveness to 6-n-propylthiouracil (PROP). Phyisology and Behavior, 68: 691-697.

Karmakar, P., Arora, R., Patel, C., Sarvaiya, B., Singh, A. \& Patel, M. 2016. Caries risk in children of Udaipur City, India using genetic taste sensitivity to 6-n-propylthiouracil. Journal of International Society of Preventive \& Community Dentistry, 6(6): 523-528.

Keller, K.L. \& Tepper, B.J. 2004. Inherited taste sensitivity to 6-n- propylthiouracil in Diet and body weight in children. Obesity Research, 12: 904-912.

Kim, U.K., Jogerson, E., Coon, H., Leppert, M., Risch, N. \& Drayna, D. 2003. Positional cloning of the human quantitative trait locus underlying taste sensitivity to phenylthiocarbamide. Science, 299: 1221-225.

Lanfer, A., Bammann, K., Knof, K., Buchecker, K., Russo, P., Veidebaum, T., Kourides, Y., de Henauw, S., Molnar, D., Bel-Serrat, S., Lissner, L. \& Ahrens, W. 2013. Predictors and correlates of taste preferences in European children: The IDEFICS study. Journal of Food Quality and Preference, 27: 128-136.

Mattes, R.D. 2004. 6-n-propylthiouracil taster status: dietary modifier, marker or misleader? In: Genetic Variation in Taste Sensitivity. J. Prescott and B.J. Tepper (Eds.). New York, Marcel Dekker. pp. 229-250.
Norliza-Ahmad, Afiq-MD Zuki, M., Azilah-Azahar, N., Han-Khor, B. \& Sakdiah-Minhat, H. 2019. Prevalence and factors associated with sugarsweetened beverage intake among undergraduate students in a public university in Malaysia. Pakistan Journal of Nutrition, 18(4): 354-363.

Ooi, S.X., Lee, P.L., Law, H.Y. \& Say, Y.H. 2010. Bitter receptor gene (TAS2R38) P49A genotypes and their association with aversion to vegetables and sweet/fat foods in Malaysian subjects. Asia Pacific Journal of Clinical Nutrition, 19(4): 491-498.

Pasquet, P., Oberti, B., Ati, J.E. \& Hladik, C.M. 2002. Relationships between threshold-based PROP sensitivities and food preferences of Tunisians. Appetite, 39(2): 167-173.

Tepper, B.J. 2008. Nutritional implication of genetic taste variation. The role of PROP sensitivity and other taste phenotype. Annual Review of Nutrition, 28: 367-388.

Tepper, B.J., White, E.A., Koelliker, Y., Lanzaro, C., d'Adamo, P. \& Gasparin, P. 2009. Genetic variation in taste in taste sensitivity to 6-npropylthiouracil and its relationship to taste perception and food selection. Annals of the New York Academy of Sciences, 1170: 126-139.

Törnwall, O., Silventoinen, K., Keskitalo-Vuokko, K., Perola, M., Kaprio, J. \& Tuorila, H. 2012. Genetic contribution to sour taste preference. Appetite, 58(2): 687-694. 
